\title{
Surgical capping of superior semicircular canal dehiscence
}

\author{
S. A. Mueller $\cdot$ D. Vibert $\cdot$ R. Haeusler $\cdot$ \\ A. Raabe $\cdot$ M. Caversaccio
}

Received: 24 January 2013/Accepted: 24 April 2013/Published online: 3 May 2013

(C) Springer-Verlag Berlin Heidelberg 2013

\begin{abstract}
Surgical plugging and resurfacing are well established treatments of superior semicircular canal dehiscence, while capping with hydroxyapatite cement has been little discussed in literature. The aim of this study was to prove the efficacy of the capping technique. Charts of patients diagnosed with superior semicircular canal dehiscence were reviewed retrospectively. All patients answered the dizziness handicap inventory, a survey analyzing the impact of their symptoms on their quality of life. Capping of the dehiscent canal was performed via the middle fossa approach in all cases. Ten out of 22 patients diagnosed with superior semicircular canal dehiscence were treated with surgical capping, nine of which were included in this study. No major perioperative complications occurred. In 8 out of $9(89 \%)$ patients, capping led to a satisfying reduction of the main symptoms. One patient underwent revision surgery 1 year after the initial intervention. Scores in the dizziness handicap inventory were lower in the surgically treated group than in the non-surgically treated group, but results were not statistically significant $(P=0.45)$. Overall, capping is a safe and efficient alternative to plugging and resurfacing of superior semicircular canal dehiscence.
\end{abstract}

Keywords Superior semicircular canal dehiscence . Canal plugging - Canal resurfacing - Canal capping .

Middle fossa approach

S. A. Mueller · D. Vibert · R. Haeusler · M. Caversaccio $(\bowtie)$ University Clinic of ENT Head and Neck Surgery,

Inselspital, Berne, Switzerland

e-mail: marco.caversaccio@insel.ch

A. Raabe

University Clinic of Neurosurgery, Inselspital, Berne,

Switzerland

\section{Introduction}

Superior semicircular canal dehiscence (SSCD) was first described by Minor in 1998 [1]. An opening in the thin bone margin of the superior semicircular canal creates a direct interface between the membranous canal and the overlying dura. This opening is also called the third window, in reference to the oval and round windows, the two physiological openings of the otic capsule. The clinical manifestations of SSCD are Tullio phenomenon and Hennebert sign as well as dizziness. When exposed to either loud sounds or changes in pressure, the labyrinthine membrane bulges through the pathological opening, modifying its elasticity. This mechanism may generate an increased endolymph movement towards the dehiscence. The consequent over-deflection of the cupula causes vertigo and nystagmus. The direction of the nystagmus corresponds to the affected superior semicircular canal $[1,2]$.

Additional auditory symptoms may also occur. In some patients, pure tone audiometry may reveal an air-bone gap in low frequencies, with bone-conducted hearing thresholds below $0 \mathrm{~dB}$. Rosowski et al. [3] suggested that because of the increased elasticity of the membranous labyrinth, acoustic energy is lost, resulting in a conductive hearing loss within the inner ear. In the past, this conductive hearing loss has often been misinterpreted as otosclerosis and has led to unnecessary and unsuccessful stapedotomies. The increased elasticity of the inner ear may also explain the abnormally low bone-conducted hearing thresholds and autophony $[4,5]$.

The pathophysiology of SSCD remains unclear. Some authors suggest underlying developmental disorders in the fetal period as a possible etiology [6], others point to microtraumatic injuries or degeneration of the overlying bone related to age-dependent metabolic changes [7, 8]. 
Several surgical techniques have been developed to repair the SSCD. The first operations were performed through the middle fossa approach [1]. Since then, the transmastoid approach has been established and is preferred by many ear surgeons. Regardless of the surgical approach, three techniques for closing the SSDC have been described, termed plugging, resurfacing, and capping. These techniques are illustrated in Fig. 1.

In plugging, the dehiscent canal is obliterated with bone paté. Using the middle fossa approach, the material is pressed through the opening of the dehiscent canal. When the transmastoid approach is used, the osseous limits of both the ampullated and non-ampullated side are opened with a diamond burr. Bone pate fashioned with bone dust and fibrinogen sealant is then introduced into both openings and the semicircular canal occluded on both sides of the dehiscence [9].

The goal of the resurfacing technique is to cover the SSCD without occluding the canal. Bone or cartilage grafts or fascia are placed over the dehiscence to achieve a continuous separation between the membranous labyrinth and the dura. When the transmastoid approach is chosen for this procedure, the bone, cartilage or fascia is placed over the dehiscence through a bore hole in the tegmen tympani and the surgeon has no direct view of the dehiscence $[10,11]$.

Capping is a modified technique of resurfacing, and has so far been little discussed in literature. This technique uses hydroxyapatite cement to close the SSCD [12].

Resurfacing has been suggested to have higher relapse rates than plugging [2]. This was found to be caused by dislocation of the grafts placed over the dehiscence. To tackle this problem, hydroxyapatite cement is used in capping, because dislocation of the cement is less probable. The aim of this study was to prove the efficacy of surgical treatment in relieving the symptoms of SSCD using the capping technique.

\section{Materials and methods}

Between January 2004 and July 2012, 22 patients identified with SSCD were followed in our clinic. Ten of these patients were treated surgically. One patient refused the use of his data; nine patients (seven men, two women) were included in the study. All patients underwent a complete otoneurological examination including clinical vestibular examination, pure tone audiometry, brainstem evoked response audiometry (BERA), videonystagmography, and vestibular evoked myogenic potential (VEMP) testing. The diagnosis of SSCD was confirmed by means of high-resolution computed tomography (CT) of the temporal bone with image reconstruction in the planes of the semicircular canals.
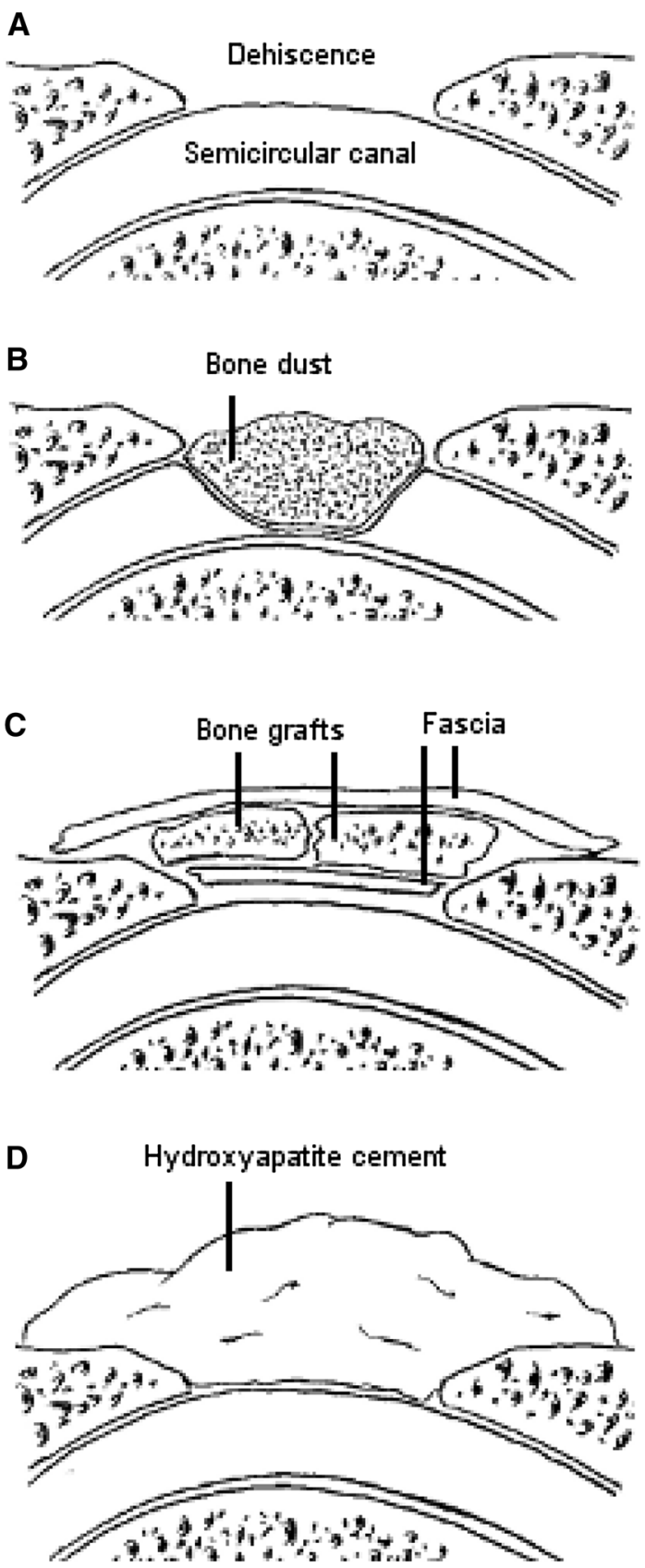

Fig. 1 Principles of the surgical repair of SSCD. a SSCD prior to surgery. b Plugging the membranous canal is compressed with bone dust. c Resurfacing the continuity of the bone overlying the membranous canal is restored with bone, cartilage or fascia. d Capping the continuity of the bone overlying the membranous canal is restored with hydroxyapatite cement

Indication for surgery

Mandatory criteria for the indication for surgery were (a) the presence of intermittent sound and/or pressure induced vertigo with Tullio phenomenon and/or Hennebert sign, verifiable by means of videonystagmography; (b) confirmation of SSCD in high-resolution CT of the 
temporal bone; and (c) absence of other possible causes of vertigo. To fortify the diagnosis of SSCD, air-bone gap in pure tone audiometry and VEMP-thresholds lower than $80 \mathrm{~dB}$ were considered. However, absence of the typical signs in these two measurements did not prevent a surgical intervention, given the above-mentioned criteria were met.

\section{Surgical technique}

All surgical interventions were performed by two of our co-authors. The middle fossa approach and capping technique were used in all cases. Craniotomy was performed in collaboration with a neurosurgeon. A CT-based navigation system (VectorVision, Brainlab, Munich, Germany) was used as an additional orientation guide in all interventions. Three types of hydroxyapatite cement were used: Norian ${ }^{\mathrm{TM}}$ (Synthes, Oberdorf, Switzerland) in six patients, BoneSource $^{\mathrm{TM}}$ (Stryker, Selzach, Switzerland) in two patients, and Hydroset ${ }^{\mathrm{TM}}$ (Stryker, Selzach, Switzerland) in one patient. A thin layer of bone dust and fibrinogen sealant was placed on the dehiscence before the cement was applied. In the first patient (patient 1), an additional bone graft was used.

\section{Follow-up}

To confirm the occlusion of the SSCD, a high-resolution $\mathrm{CT}$ of the temporal bone was obtained postoperatively for all patients (Fig. 2). After surgery, all patients were regularly re-examined by the surgeon. In addition, all patients answered a survey analyzing the impact of their symptoms on their quality of life. For this, the German adapted version of the Dizziness Handicap Inventory (DHI) [13] was used, supplemented by additional questions related to the clinical symptoms of Tullio phenomenon, Hennebert sign, autophony, and hyperacusis. The DHI was also fulfilled by the non-operated patients with SSCD.
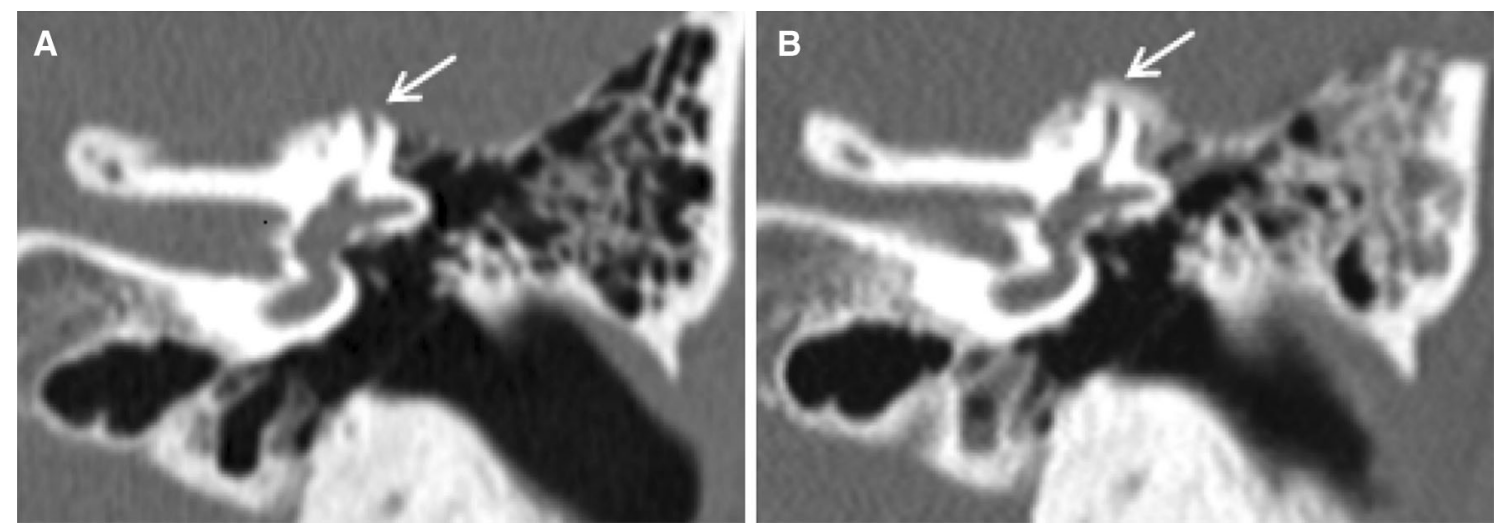

Fig. 2 High-resolution CT-scans of the temporal bone showing SSCD. a The superior semicircular canal shows an incomplete osseous lining (arrow). b After capping, hydroxyapatite cement (arrow) covers the osseous dehiscence
This study was performed in accordance with the their informed consent and have agreed to the use of their data for this study.

\section{Results and analysis}

The mean age at the time of surgery was 45.8 years (20.9-59.7). The SSCD was right sided in three cases (33\%), left sided in four (44\%), and bilateral in two (22\%). In case of bilateral SSCD, only the predominantly symptomatic side was treated surgically. Except for the implantation of a middle ear ventilation tube in patient 4 , no previous surgery had been performed on any patient. No major perioperative complications were observed. A delayed bilateral sensorineural hearing loss occurred in patient 4. Mean follow-up after surgery was 3.6 years (range $0.4-8.0$ years).

Preoperative and post-operative symptoms as well as patients' satisfaction with the outcome of the surgical treatment are listed in Table 1 . Tullio phenomenon was present in 8 out of 9 cases ( $89 \%$ ) and disappeared after surgery in all patients except patient 6 , who sometimes still experienced sound-induced vertigo when playing in his brass orchestra. Hennebert sign was present in 3 out of 9 cases $(33 \%)$ and disappeared in two cases after surgery. However, two patients reported slight pressure-induced vertigo postoperatively, even though Hennebert sign had not been documented preoperatively.

Autophony was reported by 6 out of 9 patients $(67 \%)$ and disappeared in 3 cases. Hyperacusis was reported by 7 out of 9 patients $(78 \%$ ), and disappeared in 2 cases. In pure tone audiometry, an air-bone gap $\geq 10 \mathrm{~dB}$ was measured in 7 out of 9 patients ( $78 \%$ ) before treatment. Two patients who reported hyperacusis did not have an air-bone gap $\geq 10 \mathrm{~dB}$. On the other hand, such air-bone gap was guidelines of the local ethics committee. All patients gave 
Table 1 Symptoms, overall satisfaction and DHI score of each patient

\begin{tabular}{|c|c|c|c|c|c|c|c|c|c|c|c|}
\hline \multirow[t]{2}{*}{ Patient } & \multirow[t]{2}{*}{ Ear } & \multicolumn{4}{|c|}{ Symptoms before surgery } & \multicolumn{4}{|c|}{ Symptoms after surgery } & \multirow[t]{2}{*}{ Satisfaction } & \multirow[t]{2}{*}{ DHI score } \\
\hline & & SIV & PIV & $\mathrm{AP}$ & HA & SIV & PIV & $\mathrm{AP}$ & HA & & \\
\hline Patient 1 & Left & - & + & + & - & - & + & - & - & + & 14 \\
\hline Patient 2 & Left & + & - & - & - & - & - & - & - & + & 6 \\
\hline Patient 3 & Left & + & + & + & + & - & - & - & - & + & 0 \\
\hline Patient 4 & Bilateral & + & - & + & + & - & - & + & + & - & 75 \\
\hline Patient 5 & Right & + & - & + & + & - & + & + & + & + & 32 \\
\hline Patient 6 & Right & + & + & - & + & + & - & - & + & + & 8 \\
\hline Patient 7 & Bilateral & + & - & - & + & - & + & - & + & + & 14 \\
\hline Patient 8 & Right & + & - & + & + & - & - & + & + & + & 30 \\
\hline Patient 9 & Left & + & - & + & + & - & - & - & - & + & $\begin{array}{l}44 \\
\varnothing 26.1\end{array}$ \\
\hline
\end{tabular}

SIV Sound-induced vertigo, PIV Pressure-induced vertigo, AP Autophony, HA Hyperacusis

indeed measured in the two patients who did not experience hyperacusis.

Tullio phenomenon was the main and most disturbing symptom for 7 out of 9 patients (78\%). Autophony represented the main symptom in patient 1 , while hyperacusis was the leading symptom in patient 3 . Immediately after surgery, 7 out of 9 patients $(78 \%$ ) observed the relief of their most disturbing symptom. Patient 6 observed a significant relief of his symptoms only 1 year after surgery. Patient 4 reported no improvement of his symptoms. In one case, (patient 1) revision surgery was performed because of recurrence of symptoms after 15 months, whereupon the symptoms were resolved.

Except patient 4, all patients were satisfied with the outcome of the surgical treatment (satisfaction $=89 \%$ ). Mean score of DHI was 26.1/100 points (range 0-75 points) in the surgically treated group. In the 12 patients who were not surgically treated, the mean score of DHI was $34.3 / 100$ points (range $8-82$ points). This difference between the two groups was not significant $(P=0.45)$.

\section{Discussion}

Using the capping technique through the middle fossa approach, we were able to cure all but one patient (patient 4) from their main symptoms, regardless of whether these symptoms were of auditory or vestibular origin. The surgical procedure proved safe, as no perioperative complications occurred. However, a revision surgery was performed in patient 1 because of recurrent autophony 15 months after the first operation. The second operation showed that the bone graft that had been used in this patient was partially absorbed. We therefore abandoned the use of bone grafts to repair SSCD.

The fact that hyperacusis was only improved in 5 out of 7 patients indicates that capping of SSCD has no significant effect on this symptom. However, the presence of hyperacusis was based only on patients' statements. As sound tolerance levels were not measured, it was impossible to quantify this parameter. Studies on the incidence of hyperacusis in the normal population are rare, but Andersson et al. [14] estimated the prevalence rate to be 7-9 \% in the Swedish population. In our study, $78 \%$ reported hyperacusis preoperatively, suggesting a strong relationship between this symptom and SSCD. Autophony persisted in half of the patients after surgery. However, in both patients with strong autophony and hyperacusis (patients 1 and 3) and less debilitating vestibular symptoms, autophony and hyperacusis resolved promptly after surgery.

Two patients reported non-debilitating pressure-induced vertigo after surgery, even though Hennebert sign was found negative pre and postoperatively. The significance of this finding therefore remains unclear.

Tullio phenomenon disappeared in all but one patient. Patient 6 sometimes still experienced sound-induced vertigo when playing in his brass orchestra. In patient 4 , Tullio phenomenon disappeared, but the patient continued to suffer from recurrent episodes of vertigo. She also complained of a fluctuating sensorineural hearing loss, which suggested the diagnosis of Menière's disease on the side of the symptomatic SSCD. In the context of concomitant pharmacotherapy-resistant migraine, and moderate depression, a significant aggravation of her hearing thresholds in pure tone audiometry was revealed by means of BERA testing. The origin of the vertigo symptoms remains unclear and could be associated to either the inner ear disease, the migraine or both. A somatoform component may also play a role. Either way, we assume that SSCD was not responsible for the main symptoms. Even though SSCD was symptomatic, capping was probably not the appropriate treatment in this case. 
This case demonstrates that the indication for surgery remains a great challenge. It can be difficult to distinguish the clinical manifestations of SSCD from other pathologies such as Menière's disease or otosclerosis. Moreover, though CT has $100 \%$ sensitivity in detecting SSCD, its specificity reaches only $12.5-77 \%[15,16]$. According to one study [17], a better resolution of the temporal bone can be achieved using digital volume tomography (DVT) instead of CT. However, our experiences with our DVT (Planmeca ProMax 3D Max, Planmeca Oy, Finland) do not agree with this finding. Given these technical limitations, emphasis has to be placed on the value of the clinical examination. Given the fact that surgical occlusion of an SSCD bears risks reaching as far as hearing loss, the indication for surgery must be carefully evaluated. The degree of physical and psychological disability has to be particularly taken into account. Absolute contraindications include severe hearing loss on the contralateral ear and low degree of disability. Relative contraindications are advanced age, severe comorbidities, and other possible causes of the symptoms.

Evaluation of quality of life after capping of the SSCD showed no significant difference compared to the nonsurgically treated group. It has been showed that DHI scores are highly variable in patients with SSCD [18]. Since the level of disability is an important selection criterion for surgical treatment, it is conceivable the surgically treated patients had initially more debilitating symptoms and a selection bias is probable. DHI scores were lower in the surgically treated group. This may suggest an improved quality of life in surgically treated patients, even though the results are not significant.

Transmastoid or middle fossa approach?

Because most ear surgeons are more familiar with the anatomy of the mastoid than the middle fossa, the transmastoid approach has become increasingly popular. Indeed, craniotomy can be avoided with this approach, but when the resurfacing technique is used, the middle fossa is nevertheless opened. The transmastoid plugging technique described by Beyea et al. [9] is the only technique that avoids an opening of the middle fossa altogether.

When resurfacing is performed through the transmastoid approach, exposure of the dehiscence with the mirror is sometimes impossible [19] and the correct placement of the bone, cartilage or fascia material can only be verified postoperatively by means of CT. The middle fossa approach offers a direct visualization of the dehiscence and the correct placement of the grafts is easier. In SSCD, concomitant defects in the tegmen tympani are common $[20,21]$. The middle fossa approach allows easy obliteration of these additional defects in the same session.
Concerning the complication rates of both approaches, a statistically valid comparison is difficult to achieve because of the very limited cases reported in literature [22].

The risk of sensorineural hearing loss appears to increase in revision surgery via the middle fossa approach [23]. In case of manipulation, scar tissue or adhesions of the membranous canal may facilitate a rupture of the membrane and endolymph leaking, resulting in hearing loss. Fortunately, the patient in our study who underwent revision surgery was spared from this complication. However, transmastoid plugging as described by Beyea et al. [9] may be safer for such a revision procedure, as the critical interface between the membranous labyrinth and the dura is not touched. So far, no studies addressing this problem are found in the literature.

Plugging, resurfacing or capping?

The failures of resurfacing reported in literature are mainly due to the dislocation or absorption of the graft material, as happened in our first operated patient. As hydroxyapatite cement is not absorbed and dislocation is less probable, capping may reduce this risk. Hydroxyapatite cement is widely used in neurosurgery, e.g., for cranioplasty. The most important long-term complication of cranioplasty using hydroxyapatite cement is local infection, especially when the cement is in contact with the frontal sinus. Seroma and foreign body reactions have also been reported $[24,25]$. None of these complications were seen in our study population. We consider the risk of infection to be lower than in cranioplasty, since the cement lies within the skull and is well shielded from the skin incision. To our knowledge, the effect of direct contact of the hydroxyapatite cement with the membranous labyrinth has not been studied. Because of this uncertainty, we applied a thin layer of bone dust to the membranous canal before capping of the SSCD. We observed that hardening of the cement upon application was sometimes delayed when the area of application was not absolutely dry. This may be a possible weak point of the capping technique.

In accordance with other authors [23], we consider the risk of rupture of the membranous canal to be higher in plugging than in resurfacing and capping, since manipulation of the membranous canal is more violent in plugging. On the other hand, several authors consider plugging to be more effective than resurfacing in reducing symptoms of SSCD [2, 26], because of the risk of dislocation or absorption of the graft material used in the resurfacing technique. By using a non-absorbable material in capping, we expect that this disadvantage may be neutralized. However, yet, the number of patients treated with the capping technique is too small to allow a statistical comparison of the efficacy of the three techniques. 


\section{Conclusion}

The capping technique through a middle fossa approach is a safe alternative to plugging and resurfacing in surgical treatment of SSCD. While most patients are relieved of Tullio phenomenon, hyperacusis and autophony remain more often unchanged. The success of the surgical repair of SSCD depends mainly on the correct indication, particularly in case of other concomitant pathologies that may mimic the symptoms of SSCD.

Conflict of interest The authors declare that they have no conflict of interest.

\section{References}

1. Minor LB, Solomon D, Zinreich JS, Zee DS (1998) Sound- and/ or pressure-induced vertigo due to bone dehiscence of the superior semicircular canal. Arch Otolaryngol Head Neck Surg 124:249-258

2. Minor LB (2005) Clinical manifestations of superior semicircular canal dehiscence. Laryngoscope 115:1717-1727

3. Rosowski JJ, Songer JE, Nakajima HH, Brinsko KM, Merchant SN (2004) Clinical, experimental, and theoretical investigations of the effect of superior semicircular canal dehiscence on hearing mechanisms. Otol Neurotol 25:323-332

4. Mikulec AA, McKenna MJ, Ramsey MJ, Rosowski JJ, Herrmann BS, Rauch SD, Curtin HD, Merchant SN (2004) Superior semicircular canal dehiscence presenting as conductive hearing loss without vertigo. Otol Neurotol 25:121-129

5. Minor LB, Carey JP, Cremer PD, Lustig LR, Streubel SO, Ruckenstein MJ (2003) Dehiscence of bone overlying the superior canal as a cause of apparent conductive hearing loss. Otol Neurotol 24:270-278

6. Takahashi N, Tsunoda A, Shirakura S, Kitamura K (2012) Anatomical feature of the middle cranial fossa in fetal periods: possible etiology of superior canal dehiscence syndrome. Acta Otolaryngol 132:385-390

7. Teixido M, Kung B, Rosowski JJ, Merchant SN (2012) Histopathology of the temporal bone in a case of superior canal dehiscence syndrome. Ann Otol Rhinol Laryngol 121:7-12

8. Nadgir RN, Ozonoff A, Devaiah AK, Haldermann AA, Sakai O (2011) Superior semicircular canal dehiscence: congenital or acquired condition? Am J Neuroradiol 32:947-949

9. Beyea JA, Agrawal SK, Parnes LS (2012) Transmastoid semicircular canal occlusion: a safe and highly effective treatment for benign paroxysmal positional vertigo and superior canal dehiscence. Laryngoscope 122:1862-1866

10. Amoodi HA, Makki MF, McNeil M, Bance M (2011) Transmastoid resurfacing of superior semicircular canal dehiscence. Laryngoscope 121:1117-1123
11. Crovetto M, Areitio E, Elexpuru J, Aguayo F (2008) Transmastoid approach for resurfacing of superior semicircular canal dehiscence. Auris Nasus Larynx 35:247-249

12. Hillman TA, Kertesz TR, Hadley K, Shelton C (2006) Reversible peripheral vestibulopathy: the treatment of superior canal dehiscence. Otolaryngol Head Neck Surg 134:431-436

13. Kurre A, van Gool CJ, Bastiaenen CH (2009) Translation, crosscultural adaptation and reliability of the german version of the dizziness handicap inventory. Otol Neurotol 30:359-367

14. Andersson G, Lindvall N, Hursti T, Carlbring P (2002) Hypersensitivity to sound (hyperacusis): a prevalence study conducted via the internet and post. Int J Audiol 41:545-554

15. Sequeira SM, Whiting BR, Shimony JS (2011) Accuracy of computed tomography detection of superior canal dehiscence. Otol Neurotol 32:1500-1505

16. Tavassolie TS, Penninger RT, Zuñiga MG, Minor LB, Carey JP (2012) Multi-slice computed tomography in the diagnosis of superior canal dehiscence: how much error, and how to minimize it? Otol Neurotol 33:215-222

17. Penninger RT, Tavassolie TS, Carey JP (2011) Cone-beam volumetric tomography for applications in the temporal bone. Otol Neurotol 32:453-460

18. Bogle JM, Lundy LB, Zapala DA, Copenhaver A (2012) Dizziness handicap after cartilage cap occlusion for superior semicircular canal dehiscence. Otol Neurotol 34:135-140

19. Agrawal SK, Parnes LS (2007) Transmastoid superior semicircular canal occlusion. Otol Neurotol 29:363-367

20. Minor LB (2000) Superior canal dehiscence syndrome. Am J Otol 21:9-19

21. El Hadi T, Sorrentino T, Calmels MN, Fraysse B, Deguine O, Marx M (2012) Spontaneous tegmen defect and semicircular canal dehiscence: same etiopathogenic entity? Otol Neurotol 33:591-594

22. Vlastarakos PV, Proikas K, Tavoulari E, Kikidis D, Maragoudakis P, Nikolopoulos T (2009) Efficacy assessment and complications of surgical treatment of superior semicircular canal dehiscence: a meta-analysis of published interventional studies. Eur Arch Otorhinolaryngol 266:177-186

23. Limb CJ, Carey PJ, Srireddy S, Minor LB (2006) Auditory function in patients with surgically treated superior semicircular canal dehiscence. Otol Neurotol 27:969-980

24. Gilardino MS, Cabiling DS, Bartlett SP (2009) Long-term follow-up experience with carbonated calcium phosphate cement (Norian) for cranioplasty in children and adults. Plast Reconstr Surg 123:983-994

25. Verret DJ, Ducic Y, Oxforf L, Smith J (2005) Hydroxyapatite cement in craniofacial reconstruction. Otolaryngol Head Neck Surg 133:897-899

26. Mikulec AA, Poe DS, McKenna MJ (2005) Operative management of superior semicircular canal dehiscence. Laryngoscope 115:501-507 